\title{
Appraising the Research Trend of Energy Utilization Problem in Cellular Network
}

\author{
Sowmya Naik P.T. \\ Assistant Professor \\ Dept of CS\&E., \\ City Engineering, Bangalore, India
}

\author{
K.N. Narasimha Murthy, PhD \\ PG Coordinator \& Head \\ Dept of CS\&E \\ Vemana Institute of Technology, Bangalore, India
}

\begin{abstract}
The dependency of the cellular or mobile network is increasing exponentially with the growth of dynamic demands of the mobile users globally. There is a huge trade-off between the energy consumption and service enhancement in present and upcoming future cellular network services and technologies. In order to cater up extensive coverage area, the energy required by the base station and its associated components needs to expend a good amount of energy. This paper has identified a problem of energy consumption over the usage of the cellular network, which the massive numbers of increasing mobile users are facing at present. Therefore, this paper performs a thorough investigation about the contribution being made by the prior researchers pertaining to address energy problems along with investigating of the existing research trends. The findings of the paper will highlights the techniques adopted by them and will also showcase the associated research gap from the existing studies.
\end{abstract}

\section{Keywords}

Cellular Network, Energy Consumption, Power Modelling, LTE.

\section{INTRODUCTION}

With the advance of telecommunication and increase of dynamic demands of the user, the areas of wireless network have increasingly witnessed a massive technological revolution in cellular network. Usage of mobile devices started gaining pace 20 years back, where there are limited mobile phone manufacturer and obviously very limited service providers to obtain network connectivity. But with the increasing pace of technological advancement in semiconductors, the prices of mobile devices slashed down and more services evolved in Indian consumer market. A recent report by KPMG [1] shows that there will be nearly 236 million users who will adopt the use of internet on their cell phones. The internet penetration of India is $19.2 \%$ in 2014, whereas the highest one of Australia (89.6\%) [1]. A report of Neilson mobile consumer market in 2013 discussed that $45 \%$ of the customer uses texting services in their smart phone, $26 \%$ in using social networking application, $17 \%$ in mobile banking, and $15 \%$ in browsing internet [1]. Another recent report presented by Internet and Mobile Association of India (IMAI) in 2015 shows that highest percentage (32\%) of user of mobile internet are college going student followed by non-working women to be the second highest mobile user [2]. Another interesting findings shows that $26 \%$ as majority of user access internet all the 7 days a week. These statistics evidently shows that there are lot of increasing usage of cellular network in recent times which demands a closer look of its scale of effectiveness. Another biggest problem in cellular network is call drop, which even doesn't meet the standard of Telecom Regulatory Authority of India (TRAI) [3]. Another bigger problem evolves with the increase of usage of Smart Phone market. Any smart phone offers better functionality to access network resources but at the cost of energy or battery. Till date, consumption of energy using services from cellular network is unsolved. A quick look into the existing reviews of various experts as well as researchers claims that there are 4 different consequence arising from energy problems in cellular network e.g. i) throughput, ii) bandwidth, iii) network performance, and iv) end-to-end delay [4]. In order to address energy efficiency for upcoming cellular network, it is essential that various upcoming technologies, energy saving protocols, cognitive radio, novel architectures etc. should be critically investigated. The cellular network consists of various telecommunication standards which are also supported by sophisticated infrastructure. Various international vendors have come up with offering of latest technologies to offer energy efficient services. However, till date no such technique has been seen which significant claims to reduce energy consumption in cellular network. Presence of noise and channel interference is another unavoidable circumstance in cellular network, which leads to significant energy depletion. Another significant problem is increasing the cell size for extensive coverage of the cell towards its users. Increasing the cell size will lead to more energy consumption from the base station, which may also degrade the communication performance of the user. The user may also undergo declining Quality-ofExperience (QoE) [6]. Hence, there are many adverse effects due to energy consumption in cellular network. In the present era, there are various researchers who have significant research contribution towards investigating the problems pertaining to energy dissipation. Therefore, this paper discusses some of the recent trends and practices adopted by the researchers in order to address the problem of energy dissipation in mobile network. Section II discusses about the essentials of cellular network where fundamental components of the network is comprehensively introduced. Section III discusses about the problem of energy consumption in cellular network elaborating about various factors responsible for energy depletion. Section IV discusses about the existing research trends followed by discussion of existing literatures in Section V. Finally, research gap is discussed in Section VI followed by summary of paper in Section VII.

\section{ESSENTIALS OF CELLULAR NETWORK}

The modern day communication is established using cellular network which is a connection established among the wireless 
nodes. It consists of receiver, transmitter, and cells where the distribution of the network takes place. The concept of cellular network is also called as mobile network and it provides various useful communication features to its users e.g. i) it offers superior network capacity management compare to single forms of transmitters as the similar frequency can be deployed for numerous links over multiple cells, ii) energy consumption owing to less energy used in mobile devices compared to other form of wireless transmission e.g. that from satellite and single transmitter, iii) it offers one of the highest coverage and connectivity spread over large geographical area.

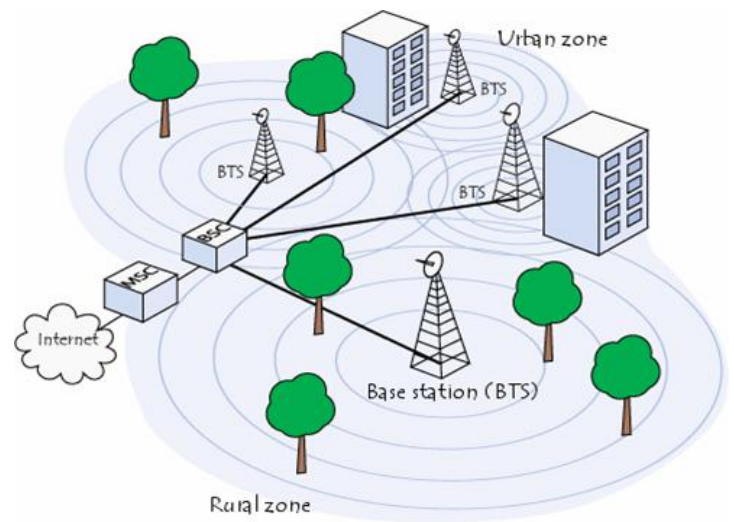

Figure 1 Coverage in Cellular Network

Fig.1 shows a typical coverage area of a cellular network, which shows that users access resources from Base Transceiver Station (BTS), which are connected through Base Station Controller (BSC). The BSC is again connected to Mobile Switching Center (MSC) that is again connected to internet. [6] The concept of cellular network involves some terminology, which we will brief in simpler way as below:

\subsection{Cell}

Cell is basically a coverage area of a base station with couple of RF channels. Normally the radius of cell ranges from 1 kilometer to 30 kilometer. The size of the cell total number of cells being used for coverage of a specific geographic area. The capacity of cell is usually determined by available bandwidth.

\subsection{Mobile Equipment}

Mobile Equipment (ME) is normally the trusted handheld device of a user used for communication using the cellular network and service provider. Different forms of ME have different supportability fo network bands and the device is identified using International Mobile Equipment identity (IMEI) number. One of the important concerns about ME in the research area is energy depletion while using the cellular network for communication. As the devices highly depend on battery to work, it dissipates significant amount of energy. The existing Smart Phone depletes more battery than legacy phones on same network.

\subsection{Base Transceiver Station}

Base Transceiver Station (BTS) is the antenna mounted in the area in order to increase the coverage and enhance the accessibility of the cellular network. Common users also know it by the name mobile tower. A typical BTS is shown in Fig.2. It is responsible for perform encoding of the multiplexing, voice packets, ciphering, etc for a radio-based signal. The coverage of single BTS is only $120^{\circ}$ of the area where it is installed. However, by mounting minimum three numbers of BTS, $360^{\circ}$ area can be covered up. Mounting of BTS is done purely based on the number of user demands as well as geographic condition of that area. BTS is identified with a code that defines location area which is covered by that particular BTS.

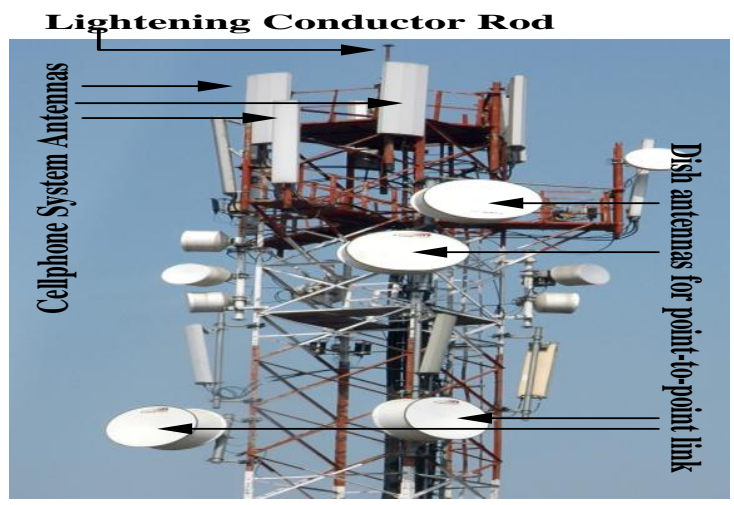

Figure 2 Base Transceiver Station

\subsection{Base Station Controller}

There could be multiple numbers of BTS for largely populated area. All the BTS are controlled by Base Station Controller (BSC) by understanding their requirements of resources of BTS. Therefore, BSC is responsible for allocation specific amount of resources (e.g. frequency, radio channel, energy, signal, etc). One of the essential operations of BSC is to assists in handover process for any user travelling from one BTS zone to another BTS zone. BSC also carry out a filtering process for minimizing the loads from all the BSC to the Mobile Switching Center (MSC) and thereby a maximal capacity of connection is established between BSC and MSC. It should be also known that BSC performs allocation of the resources from MSC itself. Therefore, BSC plays a significant role of governing the cellular network and thereby streamlining the mobile traffic. A typical BSC looks like as shown in Fig.3
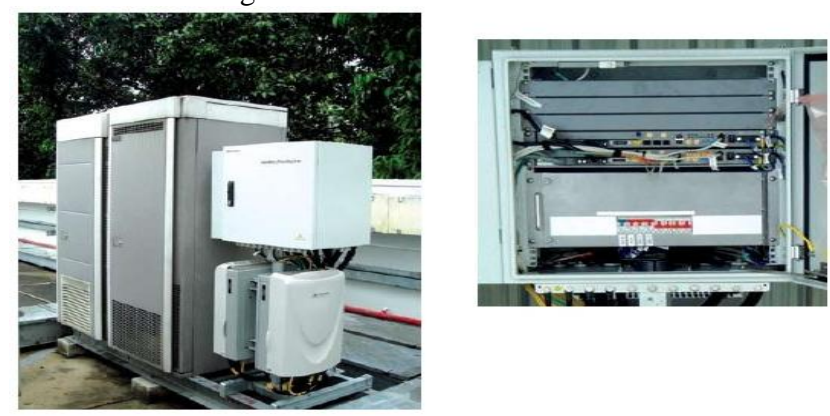

Figure 3 Huawei BTS 3036A [7]

\subsection{Mobile Switching Center}

Mobile Switching Center is one of the important actors in any mobile network. It is responsible for performing setting up of calls, routing calls, carrying out fundamental operations of switching. It also manages numerous BSC along its self connectivity with other MSC as well as other registers. Vertical handoff mechanism is largely supported by MSC. Basically, MSC is maintained in a server offering wider ranges of services. It has also the capability to control multiple forms of transport-based networks e.g. ATM, TDP, 
and IP. It can also control traffic for both WCDMA and GSM in same node. The services are managed by blade clusters that can control the power consumption to $60 \%$. A typical MSC is shown in Fig.4

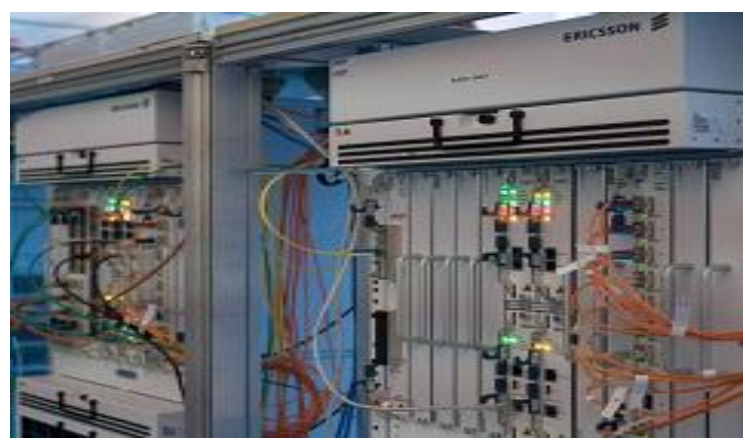

Figure 4 Erricson MSC Server [8]

Hence, all the above mentioned components are highly responsible for providing seamless communication services to the users. The services can be offer for micro cells $(<2 \mathrm{~km})$ or picocells $(<200 \mathrm{~m})$ or femtocell $(<10 \mathrm{~m})$. However, in supporting a complex mechanism of communication, there is a huge power consumption which goes almost unnoticed from the user's viewpoint. Therefore, we discuss the origin and causes of energy drainage in cellular network in next section.

\section{ENERGY CONSUMPTION IN CELLULAR NETWORK}

Energy is one of the essential factors for successful operation of cellular network. In order to undertake various significant steps for energy conservation, it is essential to mechanism a reliable technique to evaluate and thereby quantify the amount of energy being dissipated and saved in mobile networks. It is essential that monitoring of the energy consumption should be carried out by assessing both small scale and global attributes responsible for it. Such evaluation could be possible started from dense urban region to sub-urban region in order to understand the impact of dense traffic over energy consumption. It is also recommended to carry out evaluation from system perspective, which could actually give the correct measurement of Quality of service and throughput values for specific load of traffic. However, these points are all related to the possible way to find out fundamental information about energy depletion in cellular network. Now we will focus about the underlying reason behind power consumption. Our investigation found that base station consumes significantly higher amount of energy as compared to other components in cellular network discussed in prior section. We look closely into the conventional architecture of BST in Fig.5.

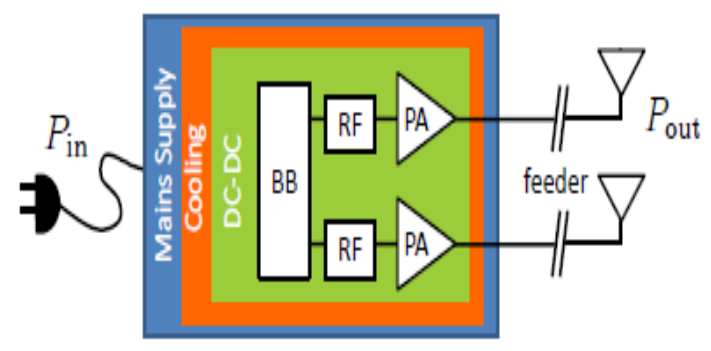

Figure 5 Architecture of BST [9]
We take the help of Fig.4 to understand the cause of energy depletion from the base station. The figure shows that a normal base station in cellular network is composed of numerous transceivers that act as single antenna to perform transmission. The transceiver will further compose of interface of baseband (BB) with both downlink and uplink section, a power amplifier (PA), and a radio frequency (RF). It will also consist of DC-DC supply of power along with a unit for establishing connection to the electrical power grid along with a cooling system [10]

\subsection{Energy Consumption due to Interface of Antenna}

The original impact of the type of antenna for ensuring an efficient usage of power can be evaluated by monitoring various loss factors sourcing from band-pass filters of antenna, feeder, duplexer, as well as matching constituent. There could be possibly an increase in the feeder loss of an antenna as sites for macro base station could be possibly positioned in different geographic coordinates. Incorporation of Remote Radio Head (RRH) can be used for overcoming the feeder loss along with PA to be used in same location. But still certain amount of energy gets dissipated spontaneously.

\subsection{Energy Consumption due to Power Amplifier}

It is said that the best power amplifier requires highest amount of output energy in order to operate efficiently. But the biggest scenario arises when the power amplifier is bound to operate in linear region owing to various multiple carrier modulation techniques (e.g. OFDM) with variable signals as well as non-linear effect of signals. This scenario assist the cellular network to resists various forms of inference due to distortion arising from non-linear signal effects. This mechanism also assists in resisting receiver's performance declination. However, this principle also gives rise to all problems of inferior energy efficiency of power amplifier that finally results in maximized energy consumption. In the existing system, technique (like clipping) exists for enhancing the efficiency of the energy for the purpose of linearizing the power amplifier along with controlling various significant interference levels. However, such conventional technique is highly dependent on additional feedback mechanism along with extra processing requirements of signals. Hence energy dissipates due to existing operation carried out by base station.

\subsection{Energy Consumption due to Transceiver}

Normally a RF transceiver in base station consists of a transmitter as well as receiver for the downlink as well as uplink communication in cellular network. The requirement for performing blocking and the linearity of the transceiver could differ from each other based on the type of the base station in consideration as well as the type of the architecture used by the system. Macro and micro base station prefers to opt for low intermediate frequency or architecture of super heterodyne type. However, Pico or femto base station requires only architectures of zero intermediate frequency. However, there are various attributes which affects the performance of RF transceivers e.g. permissible signal-to-noise-and distortion ratio, necessary bandwidth, and quantity of an antenna required for reception and transmission. 


\subsection{Energy Consumption due to Baseband Interface}

Usually, the baseband is responsible for performing modulation and demodulation, mitigating distortion, digital up or down conversion that consists of Fast Fourier Transforms for OFDM, etc. It is also responsible for performing operations for detection of signal that also includes equalization, synchronization, and compensation of nonlinearities, channel estimation, and coding or decoding of channel. In case of the huge basebands, the digital basebands also incorporates assessment of the energy being consumed by the serial link to the backbone network. More energy is depleted due to operations using MAC and platform control. The scaling of the technology is done using the energy modelling based on the ongoing advancement of the semiconductors. Although the advancement of the semiconductors results in evolution of techniques to conserve energy but it also significant results in massive leakage of energy that goes undetected and unsolved by the existing system of base station in cellular network. Although the existing system is capable enough to minimize power reduction but it is not able to cater up the energy leakage problems in base band interface. There are also various number of attributes that have a significant impact on energy in baseband interface e.g. quantity of antenna, bandwidth, considered signal processing approach.

\subsection{Energy Consumption due to Cooling}

There is a higher requirement of power supply for incorporating cooling effect on the base station components. The base station experiences significant loss due to supply of power (DC-DC) which is linear with the energy being consumed by the components of base station. It should be known that active cooling is only associated with macro base station and is not considered for smaller base stations. Unfortunately, active cooling is not considered in remote radio head as power amplifier is cooled naturally by environmental circulation of air and if feeder loss is removed than it can permit for minimal loss of energy from power amplifier.

The conventional study considers evaluating efficiency by dividing output power with input power. For traditional base station in cellular network, the amount of energy depletion is completely dependent on the amount of load due to dynamic traffic. However, due to energy being consumed by the power amplifier, the energy scales down as per the minimizing trend of traffic. The prime reason behind this is reduction of quantity of occupied subcarriers in passive mode of communication with presence of subcarriers without any data to carry. Therefore, such form of scaling over signal essentially depends on type of base station. The energy consumption by power amplifier for macro base station is 50 $60 \%$ while that of low-powered nodes it is around $30 \%$.

The indicator for measuring the energy consumption in cellular network is expressed in the form of efficiency factor or consumption factor. The existing studies undertake two types of indicators i.e. i) power per area unit that is measured in Watt $/ \mathrm{m}^{2}$ and ii) energy per bit that is measured in Joule/bit. The first indicator is represented as the network mean power usage divided by the covered area of network. It is also used for evaluating the cumulative energy being depleted and is closely associated with emission of carbon dioxide and its footprints. The attribute of power per area unit is normally applicable for lower load of traffic as the coverage of such network is limited inspite of capacity limitation. As the area of coverage is a constant factor so this attribute keep away from the variable quotient. Another metric which is commonly used is Watt per user. The second attribute energy per bit is calculated by amount of energy being consumed during a specific period of observation time divided by cumulative amount of delivered data packets in the cellular network.

Therefore, dissipating energy if conserved effectively could be productively used for enhancing the quality of features and services in the existing cellular network. Energy dissipation control is an extremely challenging task encountered by huge number of researchers from last decades. The next section discusses about research trends on such issues.

\section{EXISTING RESEARCH TRENDS}

Studies towards energy issues is one of the most visited topic of research. In order to understand the real trend, we filtered the journals published during $2010-2015$ by some of the most reknown publishers e.g. IEEE Xplore, Elsevier, Springer, and ACM Digital Library. Our investigation shows that in last 5 years, researchers have continuously addressed the issues pertaining to handoff problems, resource allocation, bandwidth, frequency reuse, energy, and security. The trend shows that energy and resource allocation are the most popular topic of research.

A better visualization of research trend could be also understood by reviewing some of the existing survey papers discussion about energy issues in cellular network. In 2011, Hasan et al. [11] have discussed about various factors that directly affects energy consumption e.g. architecture, network planning, enabling technologies etc. The authors have also reviewed some of the standard architectures of base station responsible for energy conservation. Same year, Wang et al. [12] have also reviewed some of the factors that posses as challenges in mobile networks in terms of energy issues.

In 2012, Murthy and Kavitha [13] have published a review paper discussing about energy consumption issues in cellular network. The authors have also discussed about hardware designs of the base station with respect to energy consumption. The paper have also briefly discussed about the protocols for power conservation in cellular network. In 2013, Domenico et al. [14] have presented a review paper, which have theoretical description of 3GPP LTE networks along with discussion of various energy consumption models in it. One of the significant outcomes of the study is that the much hyped LTE network is actually not available for all the forms of base station. This problem definitely highlights that power consumption is more significant for higher generation of mobile network (3G, 4G, 5G). Same year, Taruna et al. [15] have also formed the similar line of review. The authors have reviewed benefits of 10 previous research work claimed to be energy efficient.

In 2014, Ismail et al. [16] have carried out a study to review effectiveness of various services from network operators. The paper has also presented a theoretical discussion of various throughput model, power consumption model, energy and efficiency model. The paper has also reviewed some of the existing practices of traffic modelling e.g. spatial fluctuation model and temporal fluctuation model. Various review of 
scheduling approaches for energy efficiency in mobile network is also discussed.

In 2015, Weston and Ohangaa [17] have reviewed some of the energy preservation techniques considering from base station viewpoint in cellular network. Authors have also reviewed some of the prior techniques for switching base station on and off. Therefore, it has been seen there are various research work being carried out considering energy problems in cellular network. The trend highlighted in Fig.1 shows that there are around 5886 journals being published in reputed journals e.g. ACM, Elsevier, Springer, and IEEE. Our searches for existing work have excluded journals from all the other publishers. Hence roughly, it could be somewhere around 7-9,000 research manuscripts published all together during 2010-2015. The matter of worry is even after such massive research, there is no evidence that search for energy efficient communication protocol in cellular network is over. In the recent time, there are many evolution of upcoming technologies e.g. reconfigurable network, cloud, Internet-ofThings etc, which is also studied in integration with cellular network thereby increasing complexity to multi-folds. Hence, it is still an open question if how much effective is research attempts towards energy efficiency. The next section discusses about the existing research contribution towards energy issues.

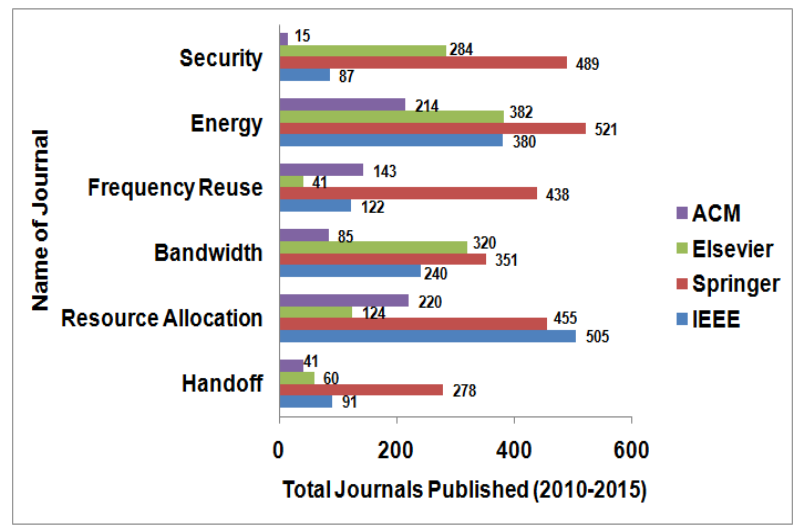

Figure 6 Existing Research Trends in Cellular Network (2010-2015)

\section{EXISTING TECHNIQUES}

This section discusses about the existing literatures where a deliberate attempt has been made to implement energy efficient techniques towards cellular network. However, different authors have different approaches to address the energy problems associated with cellular network. A closer look into the IEEE Xplore digital library shows there are 28, 293 conference papers, 6502 Journals, 221 early access articles, 75 e-books on cellular network published within the year 2005-2015. Manuscripts related to energy issues published in the same range of years are around 2000. Although, the scores mentioned are approximately relevant owing to keyword based search and real implementation work is $50 \%$ less than numbers mentioned here. Hence, we filtered out the most relevant papers in last 5 years to understand the contributions being made towards energy efficiency in cellular network.

Our first paper to be reviewed was the work done by Deng et al. [18] in 2010. The authors have introduced an analytical modelling using Poisson process in order to investigate the amount of the energy conservation from the downlink cellular network with relays. The study has considered probability, SINR, and energy as the performance parameters. The study has a good mathematical background to support the theory. Emphasis of energy problems was also seen in the study of $\mathrm{Oh}$ et al. [19]. Considering the case study of cellular data from Manchester, the study has investigated various energy related issues. The authors added that dropping probability, delay, throughput etc. are some of the important performance parameters that should be considered in assessing the cellular network performance. Tombaz et al. [20] have presented an analytical framework to investigate the variation in transmittance power in cellular network. The study considered noise and interference for developing a framework of power consumption. Another significant study was presented by Lorincz et al. [21] where a technique is shown to control the energy level of base station as per the demands of the traffic. This is one of the frequently practiced methods which is based on the principle that base station can tune up its capacity according to the fluctuation observed in traffic. Similar line of research work is also carried out by Tombaz et al. [22] emphasizing on base station. The author considered the idle energy as well as backhaul energy into consideration. In 2014, a study similar to Deng et al. [18] has been re-introduced by $\mathrm{Yu}$ et al. [23]. The study was focused on the macro base station in order to enhance the probability of coverage. The significant finding of this work is that there is potential tradeoff between the power level of macro base station and energy factor. The study also suggests that network traffic is potential factor that influence energy efficiency. Similar problem has also been focused by Esmaeilifard and Rahbar [24], who laid emphasis on complex transmission over cellular traffic. The study has presented an analytical modelling for ensuring maximal energy conservation and efficient network capacity. Study towards energy conservation is also seen in the work of Huang et al. [25] considering the case study of railway communication system. The technique was based on divide and conquers where base station is arranged on the track ways in order to assist in seamless bidirectional call handling. The numerical outcome of the study was found to posses around $20-35 \%$ of energy conservation. Most recently, the work carried out by Xiang et al. [26] have introduced a topologybased mechanism to conserve energy in cellular network. The prime aim of the study was to minimize the switching frequency of the base station considering the Long-Term Evolution (LTE) network.

The existing literature has various method and focus of issue although the core issue was always energy. Hence, we classify the discussion of existing approaches to mitigate energy issue with some of the most frequently exercised techniques as discussed below.

\subsection{Studies towards Heterogeneous Network}

Majority of the studies done towards cellular network are focused on homogeneous form. However, in order to meet the dynamic demands of communication, the base station need to perform dynamic operation and therefore require a most challenging mechanism of patterns of antenna, levels of power, connectivity etc. Domenico et al. [27] have considered the case study of femtocell in heterogeneous network and implemented an adaptive method for enhancing the energyefficient communication. Study towards heterogeneous network is also carried out by Guo and Farrell [28] in 2012 
considering the case of underlying communication principle of 4G/OFDMA network. The study is more inclined to understand effectiveness of small cell scheme with relaybased scheme. The study have also used decode and forward relay mechanism and the outcomes of the study was accessed using power consumption factor, throughput, etc. Similar direction of the study was also carried out by Lorincz and Matijevic [29], where the study have emphasized on two parameters of energy (energy per unit area and energy per bit unit area) over heterogeneous network. Soh et al. [30] have implemented a probabilistic technique and stochastic approach to understand the energy efficiency in both homo/heterogeneous cellular network. The outcome of the study was assessed using coverage probability and energy efficiency factor on multiple environments.

Similar focus on coverage was also emphasized by the work of Zhang et al. [31]. The authors have studied small cells and macro cells for multiple deployment of spectrum. The study conducted by Guo et al. [32] has used involuntary forecasting method on low powered nodes in order to understand the states of power depletion in heaver network load. Wang et al. [33] have presented a study for confirming the energy efficiency towards multimedia delivery considering network interference. The study has mainly emphasized on the scheduling technique to leverage the throughput of the cellular network. Study towards heterogeneous network was also carried out by Sambo et al. [34] where the authors have presented 2-tier deployment of transmission for minimizing the energy depletion. The outcome of the study was assessed using transmit power and downlink power depleted, and backhaul energy. Adoption of stochastic geometry is frequently seen to be used by various researchers. Panahi and Ohtsuki [35] have presented a scheme for cognitive network of heterogeneous type. The outcome of the study was assessed using outage probability and throughput.

The recent work carried out by Taranetz [36] has focused on analyzing interference. The authors have also used stochastic geometry for designing the heterogeneous cellular network. Studies conducted by Fan et al. [37] have presented a technique that ensures a sleep scheduling algorithm for heterogeneous cellular network. The outcome of the study was assessed using user capacity, energy consumption, and coverage probability. The result shows better accomplishment of coverage as well as energy conservation. The common findings of the study is that usage of stochastic geometry is the frequently used technique to design heterogeneous cellular network, where majority of the algorithms are focused on coverage probability as well as the energy efficiency.

\subsection{Study towards Quality-of-Service (QoS)}

Quality-of-Service (QoS) plays a significant parameter in leveraging the performance of cellular network. It bears a strong relationship with energy dissipation as their parameter (e.g. throughput, delay, bit rate etc) significantly affects the communication performance of cellular network. Significant study towards QoS was seen in the work of Soh et al. [38] in 2003. The study has introduced a prediction scheme for the cellular network using dynamic reservation process. Song et al. [39] have presented a technique that emphasizes on precise allocation of resource in cellular network. The study has proposed an admission scheme for joint data and voice packet as per cellular network. Giri and Bodhe [40] have presented a technique by signifying importance of multi-agent system that can ensure better QoS in cellular network. The outcome of the study was evaluated using call blocking probability with respect to traffic load. The outcomes shows that better performance of call blocking probability can ensure better QoS which can directly contribute to energy conservation. Correa and Fernandes [41] have also emphasized about the QoS factor for better scalability, network efficiency, and Quality of Experience. Study towards QoS enhancement on heterogeneous network was carried out by Kaleem et al. [42], where the authors have aimed for enhancing the throughput for cell-edge user as well as to minimize the interference owing to allocation of dynamic bandwidth. The best part of the study is its contribution towards catering up the prioritybased dynamic allocation capabilities. The outcome shows efficiency of the scheme with better throughput and minimized loss of data packet. Mtaho and Ishengoma [43] have investigated about various essential parameters that directly influence the QoS considering the case study of cellular data from Tanzania. Memis et al. [44] have carried out the study for allocating network resources for enhancing the QoS factor in cellular network. The uniqueness in this study is MIMO which is used to enhance the data rate of the cellular network. The study has also investigated the effectiveness of cross layer scheme towards resource allocation for downlink networks. The outcome of the study shows decrease in power allocation for each link and increase in time allocation for each link. Therefore, QoS parameter plays a significant role in ensuring that resource parameters are optimally used and nodes are not overburdened in carrying out networking operation. This results in significant saving of operational energy.

\subsection{Study towardsOptimization Techniques}

Existing research work has also witnessed various optimization-based techniques in order to enhance the lifetime of the cellular network. However, different researchers have applied different techniques of optimization. From the previous sub-sections, it has been seen that various researchers have implemented relay-based approach. However, it is not mandatory that usage of relays will enhance the throughput of a cell. Ji et al. [45] have presented an optimization technique to address the issues of fixed relay points in OFDM based cellular network. The study advocates that adoption of optimization over relays enhances the throughout and compensates the loss owing to changing distance of the users. Study toward optimizing energy is also seen in the work of Miao et al. [46] has presented a scheme that performs optimization of the network inflicted with interference. The investigation is carried out using simulation study for a network with two user and cooperative user. The outcome of the study was found to enhance the throughput and minimize the interference. Yu et al. [47] have presented an optimization technique that supports node-to-node communication scheme over wireless cellular network. A single cell environment was used to evaluate the scheme where the study outcome was found to enhance the network throughout and suppress an adverse interference. Tan et al. [48] have addressed the problem of optimizing the size of a cell. The study has initially mitigated problem pertaining to multiple phases of allocation of energy followed by minimizing the energy depletion issue in cellular network. The outcome of the study was found to posses much reduction of energy consumption over varying rate. Asimakis et al. [49] have addressed an optimization problem of futuristic cellular 
network strongly associated with multicasting and broadcasting. The authors have presented an optimizer that can reduce the power during transmission stage of a single cell. The outcome is assessed using response time and power consumption. Fallgren et al. [50] have presented an optimization scheme that can perform selection of better link and perform allocation of power to simplify min-max problem in throughput for multi-cell network. Using emulator-based study, the outcomes are evaluated using throughput and power. Similar study considering dynamic cellular network has been carried out by Moustakas et al. [51] where the authors discusses about an analytical framework for optimization in presence of interference in cellular network of random type. Statnikov et al. [52] have presented a mathematical model for cellular network targeted for enhance supportability of multiple criteria. Yaremko et al. [53] have presented a technique for minimizing the dissipating power for both uplink and downlink channels in cellular network. Hu and Cao [54] have adopted $\mathrm{A}^{*}$ search algorithm for minimizing the search space for scheduling offline and online for traffic aggregation for wireless network. Similar study has also been carried out by Gaikwad and Wagh [55] with a difference of focus in LTE network. Ho et al. [56] addressed the interference problem owing to coupling between the cells in cellular network. Hence, various schemes exist for optimization techniques in cellular network.

\section{RESEARCH GAP}

This section discusses about the research gap that have been identified after reviewing the existing literatures towards addressing energy issues in cellular network. The prominent research gaps identified are:

\subsection{Few Benchmarked Studies}

Inspite of presence of more than five thousand international journals towards the problems of energy efficiency, there are few studies where outcomes were evaluated with respect to performance comparative analysis of else researchers work. Moreover, the presence of existing survey paper didn't have any identification of the best work till date. Hence, in absence of this information, it is quite a challenging task to perform benchmarking for any upcoming research implementation work. Some examples of such works absence of benchmarking are Deng et al. [18], Oh et al. [19], Tombaz et al. [20], Lorincz et al. [29], Tombaz et al. [22], Esmaeilifard and Rahbar [24], Xiang et al. [26], Domenico et al. [14], Guo and Farrell [28], Soh et al. [30] etc.

\subsection{Consideration of Impractical Assumption}

There are various authors who have presented their techniques considering certain assumption which are basically not applicable in real sense. Majority of the study have considered cases of loaded traffic situation over the cell. The similar test scenarios were also considered for result analysis. But this is quite an impractical assumption as normally the traffic will be quite dynamic and unbalanced with respect to spatial and temporal domain. Another impractical assumption is the power model which doesn't consider standard radio energy model in any of the study. A mobile node (e.g. smart phone) with futuristic hardware works on first and second order radio energy model too, which is never considered in any research work

\subsection{Few Resilient Studies toward Energy Drainage}

Majority of the existing study follow the similar pattern of mitigation techniques towards energy drainage problems. The simulation is allowed to perform over considered topology or architecture or protocol of researchers, and energy efficiency parameters are observed. There are few studies where energyaware techniques are used. Also studies towards addressing energy seepage is almost none in terms of implementation, however there are many papers which has theoretical discussion about such unsolved problems.

\subsection{Lesser Evaluation towards Computational Complexity}

The present of cloud is increasing its services in cellular network which results in usage of various future generation mobile device with enhanced features. Therefore, with the demands of size miniaturization of mobile device, the problems of optimal battery lifetime is a problem. Hence, services offered by cellular network should be energy efficiency whereas existing service provider is more focused on preserving data charges rather than preserving battery of mobile devices. The existing BTS system offers energy preservation for itself but still it couldn't effective fulfill the demands of mobile devices. We highlight this point imperatively because till date the presented studies were not discussed about their computational complexity scale. There is no discussion if the presented algorithms are computationally less complex to support all the upcoming mobile devices in terms of energy preservation in cellular network.

\section{CONCLUSION}

The technological advancement in the area of wireless network has lead to evolution of various hardware, services, and protocols that aims to support futuristic generation of wireless communication like LTE and 5G in cellular network. The research community has identified some significant problems associated with mobile networks as bandwidth issue, security issue, frequency reuse issue, energy issue, and resource allocation issues. While we have performed investigation for the fundamentals trends of cellular network and its associated services, we came across with various services and product of telecommunication which claims to offer energy effective service. However, we also came to know that energy conservation is limited only to the components e.g. MSC, BTS, BSC and not for entire cellular network or for the mobile equipments. Our investigation towards the existing research papers found that existing researcher has focused on heterogeneous networks, QoS, and optimization technique pertaining to energy issues. Although the existing literatures provided a good base for new research arena, but till date no robust and benchmarked studies have been found to be resilient against energy drainage problems in cellular network. Moreover, we have also found a significant research gap as few benchmarked studies, consideration of impractical assumptions, and few resilient studies towards energy drainage. Therefore, our future work will be in direction of addressing this research gap. We have identified three areas where we can offer solution to mitigate the problems of existing system. Our first work will be towards developing a computational framework for addressing the problem of uncertain traffic and energy problems in cellular traffic using stochastic geometry. Our second future work will be towards developing a novel architecture while our third phase of future work will to be to apply further optimization to reduce the 
amount of energy consumption.

\section{REFERENCES}

[1] H. Mallya, "With 3rd largest smartphone market in the world, India to reach 314 million mobile internet users by 2017”, An article from Your Story. Retreived from http://yourstory.com/2015/07/mobile-internet-report2015/. Accessed on 19-Nov, 2015

[2] P. Panwar, "India to cross 400 million internet users by Dec 2015: IAMAI Report", An Article from Oneindia, Retereived from http://www.oneindia.com/india/india-tocross-400-million-internet-users-by-dec-2015-iamaireport-1929570.html. Accessed on 19-Nov, 2015

[3] M.G. Arun, S. Punj, "Speak uneasy", An Article from India Today, Retreived from http://indiatoday.intoday.in/story/call-drops-airtelvodafone-idea-trai/1/451901.html. Accessed on 19-Nov, 2015

[4] A. Abraham, X. H. Jiang, V. Snasel, J-S Pan, "QoS Optimization for Multimedia Streaming using Hybrid RRm and DASs over LTE Networks", SpringerProceedings of the Second Euro-China Conference on Intelligent Data Analysis and Applications, 2015

[5] P. Fiadino, M. Schiavone, P, Casas, "Vivisecting Whatsapp in Cellular Networks: Servers, Flows, and Quality of Experience", Springer-proceedings of the 7th International Workshop on Traffic Monitoring and Analysis, Spain, 2015

[6] L. Collins, S. R. Ellis, Mobile Devices: Tools and Technologies, CRC Press, 2015

[7] http://www1.huawei.com/en/products/radioaccess/signleran/index.htm

[8] http://www.ericsson.com/ourportfolio/products/mobileswitching-center-

server?nav=productcategory002|fgb_101_189

[9] P. Baudin, Wireless Transceiver Architecture: Bridging RF and Digital Communications, John Wiley \& Sons, 2014

[10] O. Jumira, S. Zeadally, Energy Efficiency in Wireless Networks, John Wiley \& Sons, 2013

[11] Z. Hasan, H. Boostanimehr, V. K. Bhargava, "Green Cellular Networks: A Survey, Some Research Issues and Challenges", IEEE Communications Surveys \& Tutorials, vol.13. pp.524-540, 2011

[12] X. Wang, A.V. Vasilakos, M. Chen, Y. Liu, T. T. Kwon, "A Survey of Green Mobile Networks: Opportunities and Challenges", Springer Journal, Mobile Network Applications, 2011

[13] C. R Murthy, C Kavitha, "A Survey of Green Base Stations in Cellular Networks", International Journal of Computer Networks and Wireless Communications, Vol.2, No.2, April 2012

[14] A De Domenico, E. C. Strinati, and A Capone, "Enabling Green Cellular Networks: A Survey and Outlook", Elsevier-Computer Communication, vol.37, pp.5-24, 2014
[15] S. Taruna, B. Pahwa and I. Kaur, "Energy Efficient Cellular Networks: A Survey", Advance in Electronic and Electric Engineering, Vol.3, No.1 pp. 127-136, 2013

[16] M. Ismail, W. Zhuang, E. Serpedin, K. Qaraqe, "A Survey on Green Mobile Networking:From The Perspectives of Network Operators and Mobile Users", IEEE Communications Survey \& Tutorials, vol.17, Iss.3, pp.1535-1556, 2015

[17] M. Weston, O. M. Ohanga, "A Survey of Base Station Sleeping Technologies for Green Cellular Networks", International Conference on Wireless Networks, 2015

[18] N. Deng, S. Zhang, W. Zhou, and J. Zhu, "A Stochastic Geometry Approach to Energy Efficiency in RelayAssisted Cellular Networks", arXiv, 2010

[19] E Oh and B. Krishnamachari, X.Liu, Z. Niu, "Toward Dynamic Energy-Efficient Operation of Cellular Network Infrastructure", IEEE- Energy Efficiency In Communications, 2011

[20] S. Tombaz, K. W. Sung, and J. Zander, "Impact of Densification on Energy Efficiency in Wireless Access Networks", IEEE GlobeCom Workshop, pp.57-62, 2012

[21] J. Lorincz, A. Capone, and D. Begusic, "Impact of service rates and base station switching granularity on energy consumption of cellular Networks", SpringerEURASIP Journal on Wireless Communications and Networking, vol.342, 2012

[22] S. Tombaz, K. W. Sung, and J. Zander, "On Metrics and Models for Energy Efficient Design of Wireless Access Networks", IEEE Wireless Communication Letters, vol.3, Iss.6, pp.649-652, 2014

[23] H. Yu, Y. Li, M. Kountouris, X. Xu, and J. Wang, "Energy efficiency analysis of relay-assisted cellular networks", Springer- EURASIP Journal on Advances in Signal Processing, vol.32, 2014

[24] M. Esmaeilifard and A. G. Rahbar, "A High Capacity Energy Efficient Approach for Traffic Transmission in Cellular Networks", Journal of Telecommunication and Information Technology, 2015

[25] J. Huang, Z. Zhong, and H. Huo, "A dynamic energysaving strategy for green cellular railway communication network", Springer- EURASIP Journal on Wireless Communications and Networking, vol.89, 2015

[26] N. Xiang, W. Li, L. Feng, F. Zhou, P. Yu, "TopologyAware Based Energy-Saving Mechanism in Wireless Cellular Networks", IEEE International Symposium on Integrated Network Management, pp.538-544, 2015

[27] A. De Domenico, "Energy Efficient Mechanisms for Heterogeneous Cellular Networks", Doctorial Thesis of Universite De grenoble, 2012

[28] W. Guo, T. O. Farrell, "Small-Net vs. Relays in a Heterogeneous Low Energy LTE Architecture", Journal of Communications, vol. 7, no. 10, October 2012

[29] J. Lorincz and T. Matijevic, "Energy-efficiency analyses of heterogeneous macro and micro base station sites", Elsevier-Computers \& Electrical Engineering, vol.40, iss. 2,2014 
[30] Y. S. Soh, T. Q. S. Quek, M. Kountouris, H. Shin, "Energy Efficient Heterogeneous Cellular Networks", IEEE Journal on Selected Areas in Communications, vol. 31, no. 5, may 2013

[31] X. Zhang, Z. Su, Z. Yan, W. Wang, "Energy-Efficiency Study for Two-tier Heterogeneous Networks (HetNet) Under Coverage Performance Constraints", Springer Journal of Mobile Network Application, 2013

[32] W. Guo, S. Wangy, X. Chux, J. Chen, H. Song, J. Zhang, "Automated Small-Cell Deployment for Heterogeneous Cellular Networks", IEEE Communications Magazine, 2013

[33] S. Wang, W. Guo, C. Khirallah, D. Vukobratovi, and J. Thompson, "Interference Allocation Scheduler for Green Multimedia Delivery", IEEE Transactions on Vehicular Technology, vol.63, Iss.5, pp.2059-2070, 2014

[34] Y.A. Sambo, M. Z. Shakir, K. A. Qaraqe, E. Serpedin, M. A. Imran, and B. Ahmed, "Energy Efficiency Improvements in HetNets by Exploiting Device-toDevice Communications", IEEE-Proccedings of $22^{\text {nd }}$ Signal Processing Conference, pp.151-155, 2014

[35] F. H Panahi and T. Ohtsuki, "Stochastic geometry modeling and analysis of cognitive heterogeneous cellular networks", Springer- EURASIP Journal on Wireless Communications, vol.141, 2015

[36] M. Taranetz, "System Level Modeling and Evaluation of Heterogeneous Cellular Networks", Dissertation of Fakultät für Elektrotechnik und Informationstechnik, 2015

[37] S. Fan, H.Tian, and C. Sengul, "Self-optimized heterogeneous networks for energy efficiency", Springer- EURASIP Journal on Wireless Communications and Networking, vol.21, 2015

[38] W-S Soh and H. S. Kim, "QoS provisioning in cellular networks based on mobility prediction techniques", IEEE Communication Magazine, vol.41, Iss.1, pp.86-92, 2003

[39] W. Song, H. Jiang, W. Zhuang, and X. Shen, "Resource Management for QoS Support in Cellular/WLAN Interworking", IEEE Network, 2005

[40] N. Giri and S. Bodhe, "Role Of Multi Agent System For QoS Guarantee In Cellular Networks", International Journal of Distributed and Parallel Systems (IJDPS) Vol.3, No.5, September 2012

[41] P. Correa, A. C. Fernandes, "End-to-End QoS in Mobile Networks", IEEE ComSoc, 2013

[42] Z. Kaleem, B. Hui and K. Chang, "QoS priority-based dynamic frequency band allocation algorithm for load balancing and interference avoidance in 3GPP LTE HetNet", Springer- EURASIP Journal on Wireless Communications and Networking, vol.185, 2014
[43] A.B. Mtaho and F. R. Ishengoma, "Factors Affecting QoS in Tanzania Cellular Networks", arXiv, 2014

[44] M. O. Memis, O. Ercetin, O. Gurbuz and S. V. Azhari, "Resource allocation for statistical QoS guarantees in MIMO cellular networks", Springer- EURASIP Journal onWireless Communications and Networking, vol.217, 2015

[45] J. Ji and W. Yu, "Bandwidth and Routing Optimization in Wireless Cellular Networks with Relays", IEEEInternational Symposium on Modelling and Optimization in Mobile, Adhoc, and Wireless Network, pp.1-5, 2009

[46] G. Miao, N. Himayat, G. Y Li, A. T. Koc, and S. Talwar, "Interference-Aware Energy-Efficient Power Optimization", IEEE Transaction on Wireless Communication, vol.10, iss.4, pp.1323-1333, 2011

[47] C-H Yu, K. Doppler, C. B. Ribeiro, and O. Tirkkonen, "Resource Sharing Optimization for Device-to-Device Communication Underlaying Cellular Networks", IEEE Transactions on Wireless Communications, vol. 10, no. 8, August 2011

[48] T. Han, N. Ansari, “Optimizing Cell Size for Energy Saving in Cellular Networks with Hybrid Energy Supplies", IEEE Global Communication Conference, pp.5189-5193, 2012

[49] K. Asimakis, C. Bouras, V. Kokkinos and A. Papazois, "Mobility-Sensitive Power Control for MBSFN Cellular Networks", International Conference on Advanced Information Networking and Applications Workshops, 2013

[50] M. Fallgren and H. E. Oddsdóttir, G. Fodor, "An Optimization Approach to Joint Cell and Power Allocation in Multicell Networks", IEEE International Conference on Communication Workshop, pp.1-6, 2011

[51] A. L. Moustakas, P. Mertikopoulos and N. Bambos, "Power Optimization in Random Wireless Networks", arXiv, 2013

[52] R. Statnikov, J. Matusov, K. Pyankov, A. Statnikov, "Multicriteria Optimization of Cellular Networks, Scientific Research", Open Journal of Optimization, vol.2, pp.53-60, 2013

[53] O. Yaremko, B. Stryhalyuk, T. Maksymyuk, O. Lavriv, D. Kozhurov, "The optimal power control method in multiuser cellular networks", An International Quarterly Journal, Vol. 2, No. 1, pp.63-67, 2013

[54] W. Hu and G. Cao, "Energy Optimization Through Traffic Aggregation in Wireless Networks", IEEE Proceedings INFOCOM, pp.916-924, 2014

[55] V. Gaikwad, T. Wagh, "Overview of Power Optimization in LTE Network", International Journal of Computer Applications, 2015

[56] C. K. Ho, D. Yuan, L. Lei and S. Sun, "Power and Load Coupling in Cellular Networks for Energy Optimization", IEEE Transactions on Wireless Communications, vol.14, Iss.1, pp.509-519, 2015 